\title{
Proximate composition, texture analysis and sensory evaluation of keropok lekor formulated with herbs and spices
}

\author{
${ }^{1}$ Zim, A.F.M.I.U., ${ }^{1}$ Akter, A., ${ }^{1,}{ }^{*}$ Ali, M.S., ${ }^{1}$ Anik, W.A., ${ }^{1}$ Ahmed, S. and ${ }^{2}$ Zamri, A.I.B. \\ ${ }^{1}$ Faculty of Food Science and Technology, Chittagong Veterinary and Animal Sciences University, Khulshi, \\ Chittagong 4225, Bangladesh \\ ${ }^{2}$ School of Food Science and Technology, Universiti Malaysia Terengganu, T145, 21300 Kuala \\ Terengganu, Terengganu, Malaysia
}

\section{Article history:}

Received: 8 March 2019

Received in revised form: 6

April 2019

Accepted: 6 April 2019

Available Online: 5 May

2019

\section{Keywords:}

Keropok Lekor,

Product Development,

Sensory evaluation,

Proximate composition

\section{DOI:}

https://doi.org/10.26656/fr.2017.3(6).050

\begin{abstract}
Keropok lekor a fish-based sausage is a popular traditional food item in Terengganu state, Malaysia. This study aimed to develop a new flavor and taste of keropok lekor. Common spices such as cumin, coriander, cinnamon, cardamom, bay leaf, nutmeg, clove, onion, ginger, garlic were used to develop a new flavor of keropok lekor. A total of 5 samples, including two original and three developed keropok lekor samples were prepared. This study also evaluated the proximate composition of two raw samples (Original and developed). The results of the proximate analysis showed a significant difference $(p<0.05)$ in all parameters except the fiber content. Hardness, springiness, and chewiness of the samples were measured by using TA-XTplus texture analyzer. There was a significant difference in the hardness level between original and developed sample. Finally, sensory evaluation according to six attributes such as color, odors, texture, flavor, taste and overall acceptability was done by 39 panelists. A 7-point hedonic scale ranging from "dislike very much" to "like very much" was employed in sensory testing. There was a significant difference $(\mathrm{p}<0.05)$ in all the sensory parameters evaluated. The results showed that most of the respondents preferred the developed samples over the original samples. Developed fried keropok lekor with bread crumb was the most acceptable one.
\end{abstract}

\section{Introduction}

Keropok lekor is one of the most popular and delicious fish-based snack food product in Malaysia. Keropok lekor is also a nutritious food item with high protein composition and high starch component (Mohamed et al., 2008). Ikan parang (Chirocentrus dorab), ikan tamban beluru (Clupea leiogaster) and ikan selayang (Decapterus russelli) are the most commonly used fish in keropok lekor processing (Nor et al., 2010). The types of fish used to make this product could affect the product characteristics (Kaewmanee et al., 2015). Fresh fish, salt, sugar and sago flour are the main ingredients used to prepare keropok lekor (Omar et al., 2011). Though keropok lekor resembles a sausage in texture, the production procedure is not that same (Bakar, 1983).

Due to lack of profit-making value, keropok lekor may need some form of modernization in order to extend its market in the future. A recent study showed young consumers' interest in having new flavor and taste of keropok lekor (Omar et al., 2011). Food ingredients such as herbs and spices are indispensable parts of various flavor and fragrance creations. The secrets of most great cuisine rely heavily on herbs and spices (Mookherjee et al., 1989). Spices such as cloves, cinnamon, black pepper, turmeric, ginger, garlic, and onions also exhibit antioxidant properties in different food systems (Al-Jalay et al., 1987).

Therefore, the main objective of this study was to develop keropok lekor by using common Bangladeshi spices in order to add some variety in taste and flavor. Moreover, people nowadays are getting conscious of the nutritional profile of a food product. That is why this study also analyzed the difference between the proximate composition of the developed keropok lekor and the original keropok lekor.

Furthermore, the acceptance level of consumers plays a pivotal role in the development of successful food products (Tuorila, 2007). Consumer's intention to buy food largely depends on the sensory profile of a food product (Wilkinson, 2000). So, the study also aimed to evaluate the sensory acceptability of the final products. 
In addition, hardness, springiness and chewiness level of the product was also analyzed as the quality of food is also depends on the texture (Kilcast and Lewis, 1990).

\section{Materials and methods}

\subsection{Sample coding}

The overall process of this research work has been shown in Figure 1. Five different samples were prepared for this study (Figure 2). Among them sample A: Boiled keropok lekor and sample B: Fried keropok lekor were prepared by using the original recipe (fish flesh, sago flour, sugar and salt). The other three samples; sample C: Developed boiled keropok lekor, sample D: Developed keropok lekor fried with bread crumbs, sample E: Developed keropok lekor curry were prepared by adding spices.

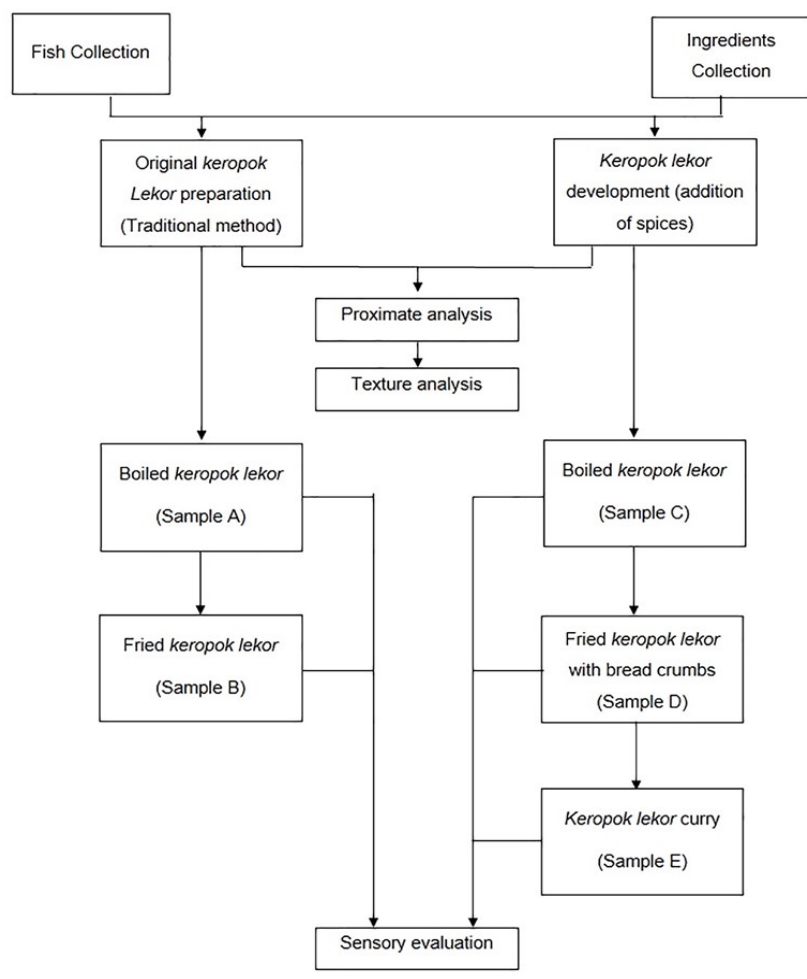

Figure 1. Flow chart of the overall process

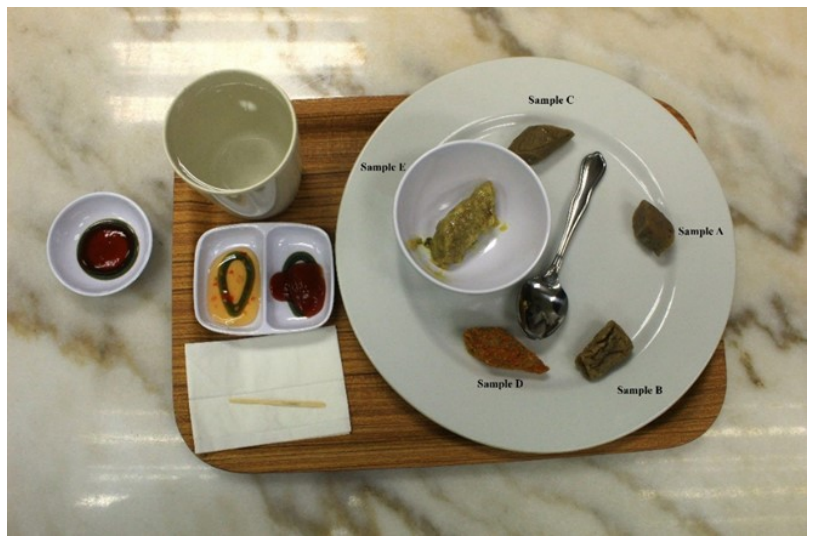

Figure 2. Five samples of keropok lekor (Sample $A=$ Boiled keropok lekor, Sample B= Fried keropok lekor, Sample C: Developed boiled keropok lekor, Sample D: Developed keropok lekor fried with bread crumb, Sample E: Developed keropok lekor curry.)

\subsection{Traditional method of preparation}

Ikan selayang (Decapterus russelli) Fish was collected from the local market of Gong badak, Kuala Terengganu. Then fishes were cleaned properly and passed through the fish deboner to prepare fish flesh. Then all ingredients (fish flesh, sago flour, salt, sugar) were mixed properly until it formed a dough. Next, the dough was shaped manually into the cylindrical roll. Each roll was shaped as $10-20 \mathrm{~cm}$ long and $2-3 \mathrm{~cm}$ in diameter. To work smoothly more flour was added from time to time. The rolls were then boiled until they are cooked. Then it was allowed to cool at room temperature. Before serving whether it was boiled (Sample A) or Fried (Sample B).

\subsection{Product development}

Product development was done by adding garlic paste, onion paste, ginger paste, green chilies and mixed spices (cumin, coriander, cinnamon, cardamom, bay leaf, nutmeg, and clove) on the dough. Roll preparation method and the amount of the main ingredients were the same as the traditional method.

\subsubsection{Sample C (developed boiled keropok lekor) preparation}

Keropok lekor rolls were immersed in the boiling water at $100^{\circ} \mathrm{C}$. The floating of the keropok lekor indicated that boiling was done. This boiled keropok lekor was the first developed sample marked as sample C.

\subsubsection{Sample D (developed keropok lekor fried with bread crumb) preparation}

At first keropok lekor rolls were boiled properly. After cooling at room temperature prepared samples were dipped into egg mixture then covered with bread crumbs. Samples were then fried in hot oil.

\subsubsection{Sample E (developed keropok lekor curry) preparation}

Twenty Rolls were boiled at first. Then they were cut into small pieces. After that, shallow frying was done by cooking rolls in oil with a depth that reached about half of the thickness of the rolls. It created a crisp and browned crust in the upper surface of the rolls. Then, sliced onion, onion paste, ginger paste, garlic paste, salt, turmeric powder and the fried keropok lekor were taken into another pan. They were mixed thoroughly. Afterward, chili and tomato sauce were added. After mixing for some time coconut milk was added into it. Then it was cooked for approximately 20 mins. Before finishing the cooking process few mint leaves, tomato slices and green chilies were added for additional flavor. 
The color of the final product was grayish yellow.

\subsection{Proximate analysis}

Proximate nutritional analysis, such as moisture, protein, fat and ash contents were determined according to the Association of Official Analytical Chemists' method (AOAC, 2012). Fiber content was determined by the fibretherm fiber analyzer (Gerhardt analytical system, Germany). The carbohydrate content was determined by subtracting the other food value i.e. Protein, Fat, Moisture, Ash, fiber. Two raw samples (original and developed) were used for this analysis. Triplicate analysis of each sample was conducted.

\subsection{Texture analysis}

Textural analysis was done by using TA-XTplus (Stable Micro Systems, UK) texture analyzer. Samples were cut into $2 \mathrm{~cm}$ thick slices. A $30 \mathrm{~kg}$ load cell was used to determine the hardness, springiness, and chewiness (Bourne, 1978). The settings were: pre-test and post-test speed of $3 \mathrm{~mm} / \mathrm{s}$, distance at $10 \mathrm{~mm}$ and time at $5 \mathrm{~s}$.

\subsection{Sensory evaluation}

Sensory evaluation of qualitative parameters (color, flavour, odor, texture, taste and overall acceptability) of the five samples was carried out using 9-point Hedonic scales (Larmond, 1977) adjusted to 7 point scale according to the following ( $7=$ like very much, $6=$ like moderately, $5=$ like slightly, $4=$ neither like nor dislike, 3 = dislike slightly, 2 = dislike moderately, $1=$ dislike very much). The five samples were presented to 39 panelists in the food sensory lab of Universiti Malaysia Terengganu.

\subsection{Statistical analysis}

The data generated were analyzed using analysis of variance (ANOVA). Tukey's post hoc test was used for the separation of means, to determine which specific groups' means were different. In case of absence of homogeneity of variance Games-Howell post hoc test was done and the Welch test was used for understanding the significance.

\section{Results and discussion}

\subsection{Proximate composition}

Proximate composition of the keropok lekor made by the original and the developed recipe showed no significant difference $(p>0.05)$ in the fiber content (Table $1)$. However, there were significant differences $(p<0.05)$ in the moisture, protein, fat, ash and carbohydrate contents of the samples (Table 1). The mean moisture content of developed keropok lekor (58.47\%) was higher than that of original keropok lekor $(56.33 \%)$. It may be due to the release of water from additional spices. Developed keropok lekor had slightly higher ash and fat contents with values of $8.90 \%$ and $1.38 \%$ as against $8.23 \%$ and $1.22 \%$ for original keropok lekor respectively. The slight increase in the ash content could be due to the addition of spices, as spices are a good source of minerals (Mehring, 1924). Besides, the low-fat content of the fish (Decapterus russelli) corroborates the lower amount of fat in both samples (Chrishanthi and Attygalle, 2013). However, the original keropok lekor had higher carbohydrate and protein contents with values of $20.81 \%$ and $12.61 \%$ as compared with $18.17 \%$ and $11.98 \%$ respectively. Protein percentage of products largely depends on the protein content of fish flesh. The amount of protein in both samples is almost similar to the study conducted by Edirisinghe et al. (2000) who showed $10.1 \%$ crude protein for Decapterus russelli. Edirisinghe et al. (2000) also reported that carbohydrate content is generally low in sea fish. Thus, it confirms that the main source of the carbohydrate was sago flour (Taewee, 2011). The fiber content varied slightly between the samples and was higher in developed keropok lekor $(1.10 \%)$ compared to original keropok lekor $(0.8 \%)$. This result indicates that the addition of spices can increase the fiber content.

Table 1. Proximate composition of keropok lekor

\begin{tabular}{ccc}
\hline \multirow{2}{*}{ Parameter $(\%)$} & \multicolumn{2}{c}{ Type of Recipe } \\
\cline { 2 - 3 } & Original & Developed \\
\hline Moisture & $56.33 \pm 0.29^{\mathrm{a}}$ & $58.47 \pm 0.22^{\mathrm{b}}$ \\
Protein & $12.61 \pm 0.09^{\mathrm{a}}$ & $11.98 \pm 0.06^{\mathrm{b}}$ \\
Fat & $1.22 \pm 0.03^{\mathrm{a}}$ & $1.38 \pm 0.01^{\mathrm{b}}$ \\
Ash & $8.23 \pm 0.03^{\mathrm{a}}$ & $8.90 \pm 0.03^{\mathrm{b}}$ \\
Fiber & $0.8 \pm 0.01^{\mathrm{a}}$ & $1.10 \pm 0.20^{\mathrm{a}}$ \\
Carbohydrate & $20.81 \pm 0.02^{\mathrm{a}}$ & $18.17 \pm 0.02^{\mathrm{b}}$ \\
\hline
\end{tabular}

Means \pm SD within the row bearing different superscripts are significantly different $(\mathrm{p}<0.05)$

\subsection{Texture}

Changes in hardness, springiness, and chewiness between two samples were shown in Table 2 . Springiness and chewiness were not significantly different $(p>0.05)$, whereas there was a significant difference $(p<0.05)$ in hardness level. From the data shown, hardness and chewiness were higher in the original keropok lekor sample than that of the developed sample. The opposite scenario was observed for the springiness. From the proximate analysis, it was found that moisture content was higher in the developed sample. This data validates the observation that higher moisture content can reduce the hardness and chewiness of a product (Huda et al., 2012). Reduced hardness level of developed keropok lekor also proved that an inverse relationship exists between hardness level and the fat 
Table 2. Texture analysis of keropok lekor

\begin{tabular}{ccc}
\hline Parameters & $\begin{array}{c}\text { Original keropok } \\
\text { lekor }\end{array}$ & $\begin{array}{c}\text { Developed keropok } \\
\text { lekor }\end{array}$ \\
\hline Hardness & $5747.770 \pm 43.888^{\mathrm{a}}$ & $4801.710 \pm 219.578^{\mathrm{b}}$ \\
Springiness & $0.919 \pm 0.020^{\mathrm{a}}$ & $0.953 \pm 0.059^{\mathrm{a}}$ \\
Chewiness & $1445.632 \pm 124.579^{\mathrm{a}}$ & $1323.881 \pm 69.400^{\mathrm{a}}$ \\
\hline
\end{tabular}

Means \pm SD within the row bearing different superscripts are significantly different $(\mathrm{p}<0.05)$

content of a product (Pietrasik, 1999).

\subsection{Sensory acceptability}

There was a significant difference $(\mathrm{p}<0.05)$ in all the sensory parameters assessed (Table 3 ). In all parameters, sample D had the highest acceptance rate. However, sample $\mathrm{C}$ scored least in all parameters, compared to the other samples. In terms of color, the highest acceptance was recorded (6.44) in sample D. It may be due to the orange color of bread crumb which made it unique among all samples. There was no significant difference between sample A and Sample C in terms of color, odor, texture, and taste. It indicates that boiled keropok lekor is almost the same whether or not spices are added. The analysis showed that sample $A$ and sample B were significantly similar in terms of flavor. But, there was a significant difference between sample C, D and E. Likewise, the score of overall acceptance followed the same trend. This suggests that keropok lekor formulated with spices did affect the flavor and overall acceptance of the products. Furthermore, the higher sensory score of flavor and taste for sample D and sample E meets one of our objectives of this study to improve the taste and flavor of keropok lekor. Spices played a significant role in this regard (Pruthi, 1980). Moreover, the sensory score of overall acceptance of the samples made it obvious that sample D was the most preferred, sample E was liked moderately, sample A and B were liked slightly and sample C was neither liked or disliked. This is an agreement with the research conducted by Omar et al. (2011) which stated that people wanted to have new taste and flavor for keropok lekor.

\section{Conclusion}

Two potential innovative products were found in this study. One was keropok lekor fried with bread crumbs and the other was keropok lekor curry. People liked the spicy flavor of keropok lekor. The orange color of bread crumb used in the developed product was captivating. It was also cheap and convenient. Addition of spices made the developed keropok lekor as a good source of minerals. The knowledge received from this study can contribute to the development of other flavored keropok lekor. Further work could focus on improving the color of original keropok lekor as well as the developed keropok lekor curry. Moreover, the use of different fish type and its effect on the overall quality of the developed keropok lekor need to be studied.

\section{Conflict of interest}

None.

\section{Acknowledgments}

The authors would gratefully like to acknowledge the laboratory and monetary support given by the University Malaysia Terengganu, Malaysia.

\section{References}

Al-Jalay, B., Blank, G., Mcconnell, B. and Al-Khayat, M. (1987). Antioxidant activity of selected spices used in fermented meat sausage. Journal of Food Protection, 50(1), 25-27. https:// doi.org/10.4315/0362-028X-50.1.25

AOAC. (2012). Official methods of analysis. 15th ed. Washington DC: Association of Official Analytical Chemists.

Bakar, J. (1983). Keropok lekor-boiling and steaming methods of processing. Pertanika Journals, 6(3), 5660 .

Bourne, M.C. (1978). Texture profile analysis. Food Technology, 32, 62-66.

Chrishanthi, E. and Attygalle, M. (2013). Fat contents and fatty acid profiles of Indian Scad. Vidyodaya Journal of Science, 16 (Abstract). Retrieved on September 10, 2018 from https://vjs.sljol.info/ articles/abstract/6032/

Edirisinghe, E.M.R.K.B., Perera, W.M.K. and

Table 3. Sensory evaluation and acceptability of keropok lekor

\begin{tabular}{lccccc}
\hline Parameters & Sample A & Sample B & Sample C & Sample D & Sample E \\
\hline Color & $5.18 \pm 1.54^{\mathrm{ab}}$ & $5.08 \pm 0.96^{\mathrm{a}}$ & $4.90 \pm 1.50^{\mathrm{a}}$ & $6.44 \pm 1.05^{\mathrm{c}}$ & $5.82 \pm 1.02^{\mathrm{bc}}$ \\
Odor & $5.13 \pm 0.83^{\mathrm{ab}}$ & $5.26 \pm 0.64^{\mathrm{b}}$ & $4.64 \pm 0.71^{\mathrm{a}}$ & $6.59 \pm 0.88^{\mathrm{c}}$ & $6.26 \pm 0.94^{\mathrm{c}}$ \\
Texture & $5.10 \pm 0.64^{\mathrm{ab}}$ & $5.36 \pm 0.63^{\mathrm{b}}$ & $4.69 \pm 0.83^{\mathrm{a}}$ & $6.59 \pm 0.79^{\mathrm{c}}$ & $5.97 \pm 0.78^{\mathrm{d}}$ \\
Flavor & $5.18 \pm 0.76^{\mathrm{b}}$ & $5.54 \pm 0.85^{\mathrm{b}}$ & $4.64 \pm 0.67^{\mathrm{a}}$ & $6.74 \pm 0.61^{\mathrm{c}}$ & $6.23 \pm 0.81^{\mathrm{d}}$ \\
Taste & $5.13 \pm 1.08^{\mathrm{ab}}$ & $5.44 \pm 0.75^{\mathrm{b}}$ & $4.64 \pm 0.96^{\mathrm{a}}$ & $6.79 \pm 0.57^{\mathrm{c}}$ & $6.00 \pm 0.92^{\mathrm{c}}$ \\
Overall Acceptance & $5.21 \pm 0.66^{\mathrm{b}}$ & $5.59 \pm 0.72^{\mathrm{b}}$ & $4.67 \pm 0.48^{\mathrm{a}}$ & $6.90 \pm 0.38^{\mathrm{c}}$ & $6.18 \pm 0.94^{\mathrm{d}}$ \\
\hline
\end{tabular}

Means \pm SD within a row with different superscripts are significantly different $(\mathrm{p}<0.05)$. Sample $\mathrm{A}=$ Boiled keropok lekor, Sample B= Fried keropok lekor, Sample C: Developed boiled keropok lekor, Sample D: Developed keropok lekor fried with bread crumb, Sample E: Developed keropok lekor curry. 
Bamunuarachchi, A. (2000). Nutritional evaluation of some small coastal fish in Sri Lanka. Journal of the National Aquatic Resources Research and Development Agency of Sri Lanka, 36, 47-53.

Huda, N., Alistair, T.L.J., Lim, H.W. and Nopianti, R. (2012). Some quality characteristics of Malaysian commercial fish sausage. Pakistan Journal of Nutrition, 11(8), 700-705. https://doi.org/10.3923/ pjn.2012.798.803

Kaewmanee, T., Karrila, T.T. and Benjakul, S. (2015). Effects of fish species on the characteristics of fish cracker. International Food Research Journal, 22(5), 2078-2087.

Kilcast, D. and Lewis, D.F. (1990). Structure and texture -their importance in food quality. Nutrition Bulletin, 15(2), 103-113. https://doi.org/10.1111/j.14673010.1990.tb00073.x

Larmond, E. (1977). Laboratory methods for sensory evaluation of food. Ottawa: Agriculture Canada, Research Branch.

Mehring, A.L. (1924). Total ash determination in spices1. Journal of Agricultural Research, 29(11), 569-574.

Mohamed, A., Jamilah, B., Abbas, K.A., Rahman, R.A. and Roselina, K. (2008). A review on physicochemical and thermorheological properties of sago starch. American Journal of Agricultural and Biological Sciences, 3(4), 639-646. https:// doi.org/10.3844/ajabssp.2008.639.646

Mookherjee, B.D., Wilson, R.A., Trenkle, R.W., Zampino, M.J. and Sands, K.P. (1989). New dimensions in flavor research: herbs and spices. In Teranishi, R., Buttery, R.G. and Shahidi, F. (Eds.). Flavor Chemistry, p. 176-187. United States of America: American Chemical Society. https:// doi.org/10.1021/bk-1989-0388.ch014

Nor, K., Loh, S.W., Zaiton, H., Jamilah, B. and Rusul, G. (2010). Quantification of coliform and Escherichia coli in Keropok lekor (Malaysian Fish Product) during processing. Journal of Applied Sciences Research, 6 (11), 1651-1655.

Omar, M., Mohd Adzahan, N., Mohd Ghazali, H., Karim, R., Abdul Halim, N.M. and Ab Karim, S. (2011). Sustaining traditional food: consumers' perceptions on physical characteristics of Keropok Lekor or fish snack. International Food Research Journal, 18(1), 117-124.

Pietrasik, Z. (1999). Effect of content of protein, fat and modified starch on binding textural characteristics, and colour of comminuted scalded sausages. Meat Science, 51(1), 17-25. https://doi.org/10.1016/S0309 $-1740(98) 00068-0$
Pruthi, J.S. (1980). Spices and condiments: chemistry, microbiology, technology. Advances in Food Research, Supplement 4, 1-449.

Taewee, T.K. (2011). Cracker" Keropok": A review on factors influencing expansion. International Food Research Journal, 18(3), 855-866.

Tuorila, H. (2007). Sensory perception as a basis of food acceptance and consumption. In Macfie, H. (Eds.). Consumer-led food product development, p. 34-65. England: Woodhead Publishing Limited. https:// doi.org/10.1533/9781845693381.1.34

Wilkinson, C., Dijksterhuis, G.B. and Minekus, M. (2000). From food structure to texture. Trends in Food Science and Technology, 11(12), 442-450. https://doi.org/10.1016/S0924-2244(01)00033-4 